\title{
Entire solutions of nonlinear differential-difference equations
}

\author{
Cuiping $\mathrm{Li}^{1}$, Feng $L \ddot{u}^{1}$ and Junfeng $X \mathrm{u}^{2^{*}}$
}

*Correspondence:

xujfmath@163.com

2 Department

of Mathematics, Wuyi

University, Jiangmen 529020,

Guangdong, People's

Republic of China

Full list of author information

is available at the end of the

article

\begin{abstract}
In this paper, we describe the properties of entire solutions of a nonlinear differentialdifference equation and a Fermat type equation, and improve several previous theorems greatly. In addition, we also deduce a uniqueness result for an entire function $f(z)$ that shares a set with its shift $f(z+c)$, which is a generalization of a result of Liu.
\end{abstract}

Keywords: Difference equation, Meromorphic function, Logarithmic order, Nevanlinna theory, Difference polynomials

Mathematics Subject Classification: 30D35, 39B12

\section{Introduction and main result}

The complex oscillation theory of meromorphic solutions of differential equations is an important topic in complex analysis. Some results can be found in Yi and Yang (2003), where Nevanlinna theory is an effective research tool. Recently, many results on meromorphic solutions of difference equations have been rapidly obtained. In this note, we are interested in the properties of entire solutions of difference and differential-difference equations.

Before proceeding, we spare the reader for a moment and assume some familiarity with the basics of Nevanlinna theory of meromorphic functions in $\mathbb{C}$ such as the first and second main theorems, and the usual notations such as the characteristic function $T(r, f)$, the proximity function $m(r, f)$ and the counting function $N(r, f) . S(r, f)$ denotes any quantity satisfying $S(r, f)=o(T(r, f))$ as $r \rightarrow \infty$, except possibly on a set of finite logarithmic measure-not necessarily the same at each occurrence. Let $a, f$ be meromorphic functions on $\mathbb{C}$. $a$ is said to be a small function of $f$ whenever $T(r, a)=S(r, f)$. S(f) denotes the family of all the small functions of $f$. $\lambda(f)$ denotes the exponent of convergence of zeros of $f, \sigma(f)$ denotes the order of $f$. A differential polynomial of $f$ means that it is a polynomial in $f$ and its derivatives with coefficients that are small functions of $f$. A differential-difference polynomial of $f$ means that it is a polynomial in $f$, its derivatives and its shifts $f(z+c)$ with coefficients that are small functions of $f$.

For a meromorphic function $f$ and a set $S \in \mathbb{C}$, we define

$$
E_{f}(S)=\bigcup_{a \in S}\{z \mid f(z)-a=0, \text { counting multiplicities }\} .
$$

(c) 2016 The Author(s). This article is distributed under the terms of the Creative Commons Attribution 4.0 International License (http://creativecommons.org/licenses/by/4.0/), which permits unrestricted use, distribution, and reproduction in any medium, provided you give appropriate credit to the original author(s) and the source, provide a link to the Creative Commons license, and indicate if changes were made. 
We say that $f$ and $g$ share a set $S$ counting multiplicities (CM), provided that $E_{f}(S)=E_{g}(S)$.

Recently, there has been a renewed interest in studying meromorphic solutions of differential-difference equations, see Peng and Chen (2013), Yang and Laine (2010) and Zhang and Liao (2011). Xu et al. (2015) considered a general differential-difference equation to obtain the following theorem.

Theorem A Consider the nonlinear differential-difference equation

$$
q(z) f^{n}(z)+a(z) f^{(k)}(z+1)=p_{1}(z) e^{q_{1}(z)}+p_{2}(z) e^{q_{2}(z)}
$$

where $p_{1}, p_{2}$ are two nonzero polynomials, $q$, a are two nonzero entire functions of finite order, $q_{1}, q_{2}$ are two nonconstant polynomials, $n \geq 2$ is an integer. Suppose that an entire function $f$ satisfies any one of the following two conditions:

(1) $\lambda(f)<\sigma(f)=\infty, \sigma_{2}(f)<\infty$;

(2) $\lambda_{2}(f)<\sigma_{2}(f)<\infty$.

Then $f$ can not be an entire solution of (1).

After studying Theorem A, we ask whether the conclusion still holds if the condition $\sigma_{2}(f)<\infty$ is omitted in (1). In the paper, we consider the problem and give an affirmative answer.

Theorem 1 Suppose that an entire function $f$ satisfies the following condition:

$$
\lambda(f)<\sigma(f)=\infty .
$$

Then $f$ can not be an entire solution of (1).

Liu (2009) used the idea of shared set (see Lü and Xu 2008) and studied the uniqueness problem of entire function $f(z)$ shares a set with its difference shift $f(z+c)$ as follows.

Theorem B Let $f$ be a transcendental entire function of finite order, $c$ is nonzero complex number, and let $a(z) \in S(f)$ be a non-vanishing periodic entire function with period c. If $f(z)$ and $f(z+c)$ share the set $\{a(z),-a(z)\} C M$, then $f(z)$ must take one of the following conclusions:

(1) $f(z) \equiv f(z+c)$

(2) $f(z)+f(z+c) \equiv 0$

(3) $f(z)=\frac{1}{2}\left(h_{1}(z)+h_{2}(z)\right)$, where $\frac{h_{1}(z+c)}{h_{1}(z)}=e^{-\gamma}, \frac{h_{2}(z+c)}{h_{2}(z)}=e^{-\gamma}$, $h_{1}(z) h_{2}(z)=a^{2}(z)\left(1-e^{-2 \gamma}\right)$ and $\gamma$ is a polynomial.

Note that the form of conclusion (3) is not similar to (1) and (2). So, it is necessary to further study the problem. In the paper, we consider Theorem B again. Due to the different method of proof we employ, we obtain the following result. 
Theorem 2 Under the conditions of Theorem $\mathrm{B}$, then

(I) $f(z) \equiv f(z+2 c)$;

(II) $f(z)+f(z+2 c) \equiv 0$.

Examples Below, we provide two examples to show that the cases (I) and (II) occur.

(a) Let $f(z)=e^{z}$ and $c=2 \pi i$. Then for any $a(z) \in S(f)$, we notice that $f(z)$ and $f(z+c)$ share $\{a(z),-a(z)\}$ and we can easily see that $f(z)=f(z+2 c)$. This example satisfies (I) of Theorem 2 .

(b) Let $f(z)=\cos z$ and $c=\frac{\pi}{2}$. Then for any $a=\frac{\sqrt{2}}{2}$, we notice that $f(z)$ and $f(z+c)$ share $\{a(z),-a(z)\}$. Furthermore, we can easily obtain $f(z)+f(z+2 c)=0$. This example satisfies case (II) of Theorem 2.

Tang and Liao (2007) considered the entire solutions of a differential equation. Liu and Cao (2013) considered a q-difference analogue of the above differential equation. Liu and Yang (2013) further generalized the result of Tang and Liao (2007) from differential equations to difference equations. They deduced the entire solutions of generalization of Fermat type equation and obtain below result.

Theorem C Let P, Q be two nonzero polynomial. If the difference equation

$$
f(z)^{2}+P(z)^{2} f(z+c)^{2}=Q(z)
$$

admits a transcendental entire solution of finite order, then $P(z) \equiv \pm 1$ and $Q(z)$ reduces to a constant $q$. Thus $f(z)=\sqrt{q} \sin (A z+B)$, where $B$ is a constant and $A=(4 \pi+1) \backslash 2 c$, where $k$ is an integer.

At the end of the paper, by considering a different proof of Theorem $\mathrm{C}$, we generalize Theorem $C$ from polynomial $P$ to small function $P$ as follows.

Theorem 3 Under the conditions of Theorem $\mathrm{C}$ and suppose that $P(z)$ is nonzero small entire function off, then the conclusions of Theorem $\mathrm{C}$ still hold.

\section{Some lemmas}

In this section, we state some results that we employ in our proofs.

Lemma 1 (Halburd and Korhonen 2006, Theorem 2.1) Let f be a meromorphic function with a finite order, and let $c$ be complex number, $\delta<1$. Then

$$
m\left(r, \frac{f(z+c)}{f(z)}\right)=o\left(\frac{T(r, f)}{r^{\delta}}\right)=S(r, f)
$$

where $S(r, f)=o(T(r, f))$ for all $r$ outside of a possible exceptional set $E$ with finite logarithmic measure. 
Lemma 2 (Yang and Laine 2010, Theorem 2.3) Let f be a transcendental entire function, $Q(z)$ is the canonical product of $f$ constructed by the zeros off. Then $\sigma(Q)=\lambda(Q)=\lambda(f)$.

The Hadamard theorem of entire functions of infinite order with $\sigma_{2}(f)<\infty$ has been proved in Jank and Volkmann (1985). In the following proof, we need to remove the condition $\sigma_{2}(f)<\infty$. Similar to the proof of the Hadamard theorem, we prove the following result.

Lemma 3 Let $f$ be an entire function of infinite order with $\lambda(f)<\infty$. Then $f$ can be represented as

$$
f(z)=Q(z) e^{g(z)}
$$

where $Q(z)$ is the canonical product of f constructed by the zeros of $f, g(z)$ is a transcendental entire function such that

$$
\lambda(Q)=\lambda(f)=\sigma(Q), \sigma(f)=\sigma\left(e^{g}\right)=\infty .
$$

Proof Let

$$
F(z)=\frac{f(z)}{Q(z)}
$$

Then $F(z)$ is entire with a Picard exceptional value 0 , and hence $F(z)=e^{g(z)}$, where $g(z)$ is an entire function.

Since $Q(z)$ is the canonical product of $f$ constructed by the zeros of $f$, then $\lambda(f)=\lambda(Q)$. By Lemma 2, we have $\sigma(Q)=\lambda(Q)=\lambda(f)<\infty$.

Note that $\sigma(Q)<\sigma(f)=\infty$, we have $\sigma\left(e^{g}\right)=\max \{\sigma(Q), \sigma(f)\}=\sigma(f)=\infty$.

\section{Proof of Theorem 1}

Suppose that $f$ is an entire solution of Eq. (1) and satisfying $\lambda(f)<\sigma(f)$. By Theorem A, it is suffice to prove Theorem 1 for the case $\sigma_{2}(f)=\infty$.

By Lemma 3, we can set

$$
f(z)=Q(z) e^{g(z)},
$$

where $Q$ is an entire function, $g$ is a transcendental entire function such that

$$
\lambda(Q)=\lambda(f)=\sigma(Q), \sigma(f)=\sigma\left(e^{g}\right)=\infty .
$$

From the condition $\lambda(f)<\infty$, we have $\sigma(Q)<\infty, \sigma_{2}(Q)=0$. So

$\sigma_{2}(f)=\max \left\{\sigma_{2}\left(e^{g}\right), \sigma_{2}(Q\}\right)=\sigma_{2}\left(e^{g}\right)=\sigma(g)=\infty$.

Substituting $f(z)=Q(z) e^{g(z)}$ into (1) we obtain that

$$
q(z) Q^{n}(z) e^{n g(z)}+a(z) H(z) e^{g(z+1)}=p_{1}(z) e^{q_{1}(z)}+p_{2}(z) e^{q_{2}(z)},
$$

where $H(z)$ is a differential polynomial in $Q(z+1)$ and $g(z+1)$.

Set $G(z)=g(z+1)-n g(z)$, then (3) becomes

$$
q(z) Q^{n}(z)+a(z) H(z) e^{G(z+1)}=e^{-n g(z)}\left(p_{1}(z) e^{q_{1}(z)}+p_{2}(z) e^{q_{2}(z)}\right)
$$


which implies

$$
q(z) Q^{n}(z)+a(z) H(z) e^{G(z+1)}-e^{-n g(z)}\left(p_{1}(z) e^{q_{1}(z)}+p_{2}(z) e^{q_{2}(z)}\right)=0 .
$$

Let $A_{1}=q(z) Q^{n}(z), A_{2}=a(z) H(z), A_{3}=p_{1}(z) e^{q_{1}(z)}+p_{2}(z) e^{q_{2}(z)}$. It is easy to see that $A_{1}$ and $A_{3}$ are of finite order. So $A_{1}$ and $A_{3}$ are two small functions of $e^{-n g}$, which means that

$$
T\left(r, A_{1}\right)=T\left(r, A_{3}\right)=S\left(r, e^{-n g}\right) .
$$

Obviously, $T(r, g)=S\left(r, e^{-n g}\right)$. Note that $H$ is a differential polynomial in $Q(z+1)$ and $g(z+1)$, so $T\left(r, A_{2}\right)=S\left(r, e^{-n g}\right)$. Rewrite (4) as

$$
A_{2} e^{G(z+1)}=A_{3} e^{-n g(z)}-A_{1} .
$$

Next we show that $A_{3} \neq 0$. Suppose $A_{3}=0$, then (1) becomes

$$
q(z) f^{n}(z)=-a(z) f^{(k)}(z+1),
$$

which implies that $n T(r, f) \leq T(r, f)+S(r, f)$, a contradiction. Thus, $A_{3} \neq 0$.

Suppose that $A_{1} \neq 0$. By using the second main theorem and (5), we have

$$
\begin{aligned}
T\left(r, e^{-n g}\right) & \leq N\left(r, e^{-n g}\right)+N\left(r, \frac{1}{e^{-n g}}\right)+N\left(r, \frac{1}{e^{-n g}-\frac{A_{1}}{A_{3}}}\right)+S\left(r, e^{-n g}\right) \\
& \leq N\left(r, \frac{1}{A_{2}}\right)+S\left(r, e^{-n g}\right) \\
& =S\left(r, e^{-n g}\right),
\end{aligned}
$$

which is a contradiction. So $A_{1}=0$, which implies $Q(z)=0$, a contradiction.

Thus, we finish the proof of Theorem 1.

\section{Proof of Theorem 2}

Since $f(z)$ is an entire function of finite order and $f(z), f(z+c)$ share the set $\{a(z),-a(z)\}$, then,

$$
\frac{(f(z+c)-a(z))(f(z+c)+a(z))}{(f(z)-a(z))(f(z)+a(z))}=e^{\alpha},
$$

where $\alpha$ is a polynomial. Since $a$ is a periodic entire function with period $c$, we infer by Lemma 1 that

$$
m\left(r, \frac{f(z+c)-a(z)}{f(z)-a(z)}\right)=S(r, f)
$$

and

$$
m\left(r, \frac{f(z+c)+a(z)}{f(z)+a(z)}\right)=S(r, f) .
$$

From (6)-(8), we obtain

$$
T\left(r, e^{\alpha}\right)=m\left(r, e^{\alpha}\right)=S(r, f) .
$$


Let $F(z)=f^{2}(z)$, then (6) can be rewritten as

$$
F(z+c)-a^{2}(z)=\left(F(z)-a^{2}(z)\right) e^{\alpha},
$$

which implies

$$
F(z+c)=e^{\alpha}\left(F(z)-a^{2}(z)\right)+a^{2}(z) .
$$

Dividing (10) with $e^{\alpha}$, we get

$$
\frac{1}{e^{\alpha}} F(z+c)=F(z)-a^{2}(z)\left(1-e^{-\alpha(z)}\right),
$$

that is, all zeros of $F(z+c)$ are the zeros of $F(z)-a^{2}(z)\left(1-e^{-\alpha(z)}\right)$. Since $F(z)$ just has multiple zeros, we have $F(z)-a^{2}(z)\left(1-e^{-\alpha(z)}\right)$ just has multiple zeros.

Rewrite (10) as

$$
e^{\alpha} F(z)=F(z+c)-a^{2}(z)\left(1-e^{\alpha(z)}\right),
$$

which implies

$$
e^{\alpha(z-c)} F(z-c)=F(z)-a^{2}(z-c)\left(1-e^{\alpha(z-c)}\right)=F(z)-a^{2}(z)\left(1-e^{\alpha(z-c)}\right) .
$$

So $F(z)-a^{2}(z)\left(1-e^{\alpha(z-c)}\right)$ has multiple zeros.

From (9)-(12), it follows that $F(z), \quad F(z)-a^{2}(z)\left(1-e^{-\alpha(z)}\right)$ and $F(z)-a^{2}(z)\left(1-e^{\alpha(z-c)}\right)$ just have multiple zeros.

Suppose that the three functions $0, a^{2}(z)\left(1-e^{-\alpha(z)}\right)$ and $a^{2}(z)\left(1-e^{\alpha(z-c)}\right)$ are distinct from each other.

By using the second main theorem, we obtain

$$
\begin{aligned}
2 T(r, F) & \leq \\
\leq & \bar{N}\left(r, \frac{1}{F}\right)+\bar{N}\left(r, \frac{1}{F-a^{2}(z)\left(1-e^{-\alpha(z)}\right)}\right)+\bar{N}\left(r, \frac{1}{F-a^{2}(z)\left(1-e^{\alpha(z-c)}\right)}\right)+S(r, F) \\
\leq & \frac{1}{2} N\left(r, \frac{1}{F}\right)+\frac{1}{2} N\left(r, \frac{1}{F-a^{2}(z)\left(1-e^{-\alpha(z)}\right)}\right)+\frac{1}{2} N\left(r, \frac{1}{F-a^{2}(z)\left(1-e^{\alpha(z-c)}\right)}\right) \\
& +S(r, F) \leq \frac{3}{2} T(r, F)+S(r, F),
\end{aligned}
$$

a contradiction.

Then two of the above three functions must be equal.

(i) If $a^{2}(z)\left(1-e^{-\alpha(z)}\right)=0$, then $e^{\alpha(z)}=1$, which implies $f(z) \equiv f(z+c)$ or $f(z)+f(z+c) \equiv 0$. Furthermore, it leads to the case (I).

(ii) If $a^{2}(z)\left(1-e^{\alpha(z-c)}\right)=0$, then $e^{\alpha(z-c)}=1$, which implies $e^{\alpha(z)}=1$, we get the same conclusion of (i).

(iii) If $a^{2}(z)\left(1-e^{-\alpha(z)}\right)=a^{2}(z)\left(1-e^{\alpha(z-c)}\right)$, then

$$
1-e^{-\alpha(z+c)}=1-e^{\alpha(z)},
$$

which implies that $1=e^{\alpha(z)+\alpha(z+c)}$. Then, a calculation leads to $\alpha$ is a constant and $e^{2 \alpha}=1$. So, $e^{\alpha}= \pm 1$.

If $e^{\alpha}=1$, then we get the same conclusion of (i) and (ii).

If $e^{\alpha}=-1$, then 
$f^{2}(z+c)-a^{2}(z)=-f^{2}(z)+a^{2}(z)$.

Furthermore,

$$
\begin{aligned}
f^{2}(z+2 c)-a^{2}(z) & =f^{2}(z+2 c)-a^{2}(z+c)=-f^{2}(z+c)+a^{2}(z+c) \\
& =-f^{2}(z+c)+a^{2}(z)=f^{2}(z)-a^{2}(z),
\end{aligned}
$$

which implies $f^{2}(z)=f^{2}(z+2 c)$. We obtain $f(z) \equiv f(z+2 c)$ or $f(z)+f(z+2 c) \equiv 0$, which is (I) or (II).

Thus, we finish the proof of Theorem 2 .

\section{Proof of Theorem 3}

Suppose that $f$ is a transcendental entire solution of finite order of (2). Set

$$
G(z)=f^{2}(z), H(z)=P^{2}(z) f^{2}(z+c),
$$

then (2) can be rewritten as $G(z)+H(z)=Q(z)$, which implies

$$
G(z)-Q(z)=-H(z) .
$$

Thus all the zeros of $H(z)$ are the zeros of $G(z)-Q(z)$. Since $H$ just has multiple zeros, $G-Q$ just has multiple zeros.

From (13), we have $H(z-c)=G(z) P^{2}(z-c)$. Then

$$
\begin{aligned}
-G(z-c)=H(z-c)-Q(z-c) & =G(z) P^{2}(z-c)-Q(z-c) \\
& =P^{2}(z-c)\left[G(z)-\frac{Q(z-c)}{P^{2}(z-c)}\right] .
\end{aligned}
$$

Note that $G(z-c)=f^{2}(z-c)$ just has multiple zeros. Then, it follows from the above equation that $G(z)-\frac{Q(z-c)}{P^{2}(z-c)}$ just has multiple zeros.

From (13)-(15), we obtain $G, G(z)-Q(z), G(z)-\frac{Q(z-c)}{P^{2}(z-c)}$ just have multiple zeros.

Suppose that the three functions $0, Q(z), \frac{Q(z-c)}{P^{2}(z-c)}$ are distinct from each other. Then, by using the second main theorem, we obtain

$$
\begin{aligned}
2 T(r, G) & \leq \bar{N}\left(r, \frac{1}{G}\right)+\bar{N}\left(r, \frac{1}{G-Q(z)}\right)+\bar{N}\left(r, \frac{1}{G-\frac{Q(z-c)}{P^{2}(z-c)}}\right)+S(r, G) \\
& \leq \frac{1}{2} N\left(r, \frac{1}{G}\right)+\frac{1}{2} N\left(r, \frac{1}{G-Q(z)}\right)+\frac{1}{2} N\left(r, \frac{1}{G-\frac{Q(z-c)}{P^{2}(z-c)}}\right)+S(r, G) \\
& \leq \frac{3}{2} T(r, G)+S(r, G),
\end{aligned}
$$

a contradiction. Then two of the above three functions must be equal.

Since $Q(z) \neq 0, P(z) \neq 0$, we have $Q(z)=\frac{Q(z-c)}{P^{2}(z-c)}$, which implies

$$
P^{2}(z) Q(z+c)=Q(z) .
$$

Because $Q(z)$ is a nonzero polynomial, we have $P^{2}(z) \equiv 1$ and $Q(z)$ reduces to a constant. Furthermore, by Liu et al. (2012, Theorem 1.1), we obtain the desired result.

Thus, we finish the proof of Theorem 3. 


\section{Conclusions}

This paper provides three results. Firstly, we consider the existence of the solutions of a nonlinear differential-difference equation under a general condition. Secondly, we prove a uniqueness theorem of entire function $f(z)$ shares a set with its difference shift $f(z+c)$. At last, we obtain the entire function solutions of a general Fermat type equation. The above three results were obtained by the different proofs, which can be used later.

\section{Authors' contributions}

$\mathrm{CPL}, \mathrm{FL}$ and JFX completed the main part of this article. All authors read and approved the final manuscript.

\section{Author details}

${ }^{1}$ College of Science, China University of Petroleum, Qingdao 266580, Shandong, People's Republic of China. ${ }^{2}$ Department of Mathematics, Wuyi University, Jiangmen 529020, Guangdong, People's Republic of China.

\section{Acknowledgements}

The research was supported by the Natural Science Foundation of Shandong Province Youth Fund Project (ZR2012AQ021), the Fundamental Research Funds for the Central Universities (15CX08011A,15CX05063A), the training plan for the Outstanding Young Teachers in Higher Education of Guangdong (Nos. Yq2013159, SYq2014002) and NSF of Guangdong Province (Nos. 2016A030313002, 2015A030313644).

\section{Competing interests}

The authors declare that they have no competing interests.

Received: 26 January 2016 Accepted: 29 April 2016

Published online: 12 May 2016

\section{References}

Halburd RG, Korhonen RJ (2006) Nevanlinna theory for the difference operator. Ann Acad Sci Fenn Math 31:463-478 Jank G, Volkmann L (1985) Meromorphe funktionen und differentialgleichungen. Birkhäuser, Basel-Boston

Liu K (2009) Meromorphic functions sharing a set with applications to difference equations. J Math Anal Appl 359:384-393

Liu K, Cao TB (2013) Entire solutions of fermat type q-difference differential equations. Electron J Differ Eq. 2013(59):1-10

Liu K, Cao TB, Cao HZ (2012) Entire solutions of Fermat type differential-difference equations. Arch Math 99:147-155

Liu K, Yang LZ (2013) On entire solutions of some differential-difference equations. Comput Methods Funct Theory 13:433-447

Lü F, Xu JF (2008) Sharing set and normal families of entire functions and their derivatives. Houst J Math 34(4):1213-1223

Peng CW, Chen ZX (2013) On a conjecture concerning some nonlinear difference equations. Bull Malays Math Sci Soc 36:221-227

Tang JF, Liao LW (2007) The transcendental meromorphic solutions of a certain type of nonlinear differential ential equations. J Math Anal Appl 334:517-527

Xu N, Cao TB, Liu K (2015) Entire solutions for nonlinear differential-difference equations. Electron J Differ Eq. 22:1-8

Yang CC, Laine I (2010) On analogies between nonlinear difference and differential equations. Proc Jpn Acad Ser A 86:10-14

Yi HX, Yang CC (2003) Uniqueness theory of meromorphic functions. Kluwer, New York

Zhang J, Liao LW (2011) On entire solutions of a certain type of nonlinear differential and difference equations. Taiwan J Math 15:2145-2157

\section{Submit your manuscript to a SpringerOpen ${ }^{\circ}$ journal and benefit from:}

- Convenient online submission

Rigorous peer review

- Immediate publication on acceptance

- Open access: articles freely available online

- High visibility within the field

- Retaining the copyright to your article

Submit your next manuscript at $>$ springeropen.com 